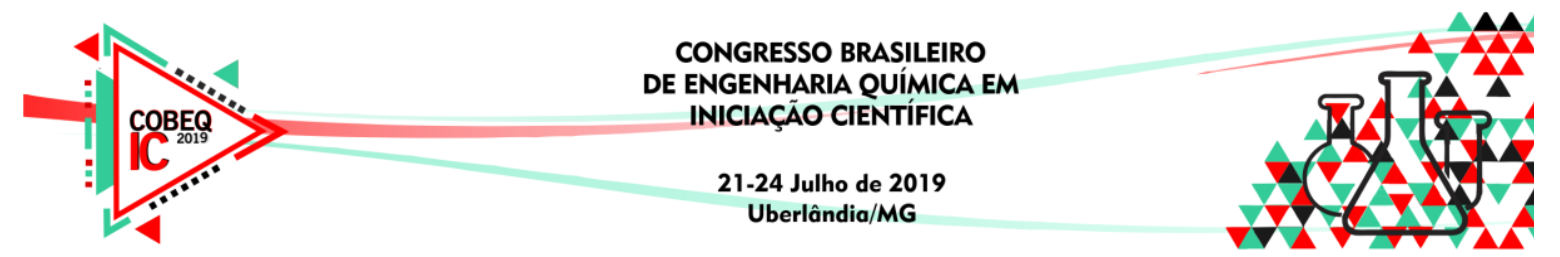

\title{
AVALIAÇÃO DO EFEITO DA TENSÃO SUPERFICIAL NO DIÂMETRO E VELOCIDADE DE BOLHAS UTILIZANDO CFD
}

\author{
H. H. JACOMINI ${ }^{1}$, J. N. M. BATISTA ${ }^{1}$ e R. BÉTTEGA ${ }^{1}$ \\ ${ }^{1}$ Universidade Federal de São Carlos, Departamento de Engenharia Química \\ E-mail para contato: hugo_jacomini@ hotmail.com
}

\begin{abstract}
RESUMO - No presente trabalho, avaliou-se a formação de bolhas de ar em um tanque de flotação empregando diferentes tensões superficiais através de testes experimentais e utilizando a fluidodinâmica computacional (Computational Fluid Dynamics - CFD). As simulações foram conduzidas utilizando o software FLUENT® 18.2. Para a verificação do modelo proposto, os dados simulados de velocidade e diâmetro de bolha foram comparados aos resultados experimentais, tanto quantitativamente quanto qualitativamente. Através da CFD foi possível inferir o comportamento e a dinâmica das bolhas para as diferentes tensões superficiais, verificando-se desvios aceitáveis entre os dados de velocidade de bolha obtidos experimentalmente e por meio de simulações. Os diâmetros das bolhas experimentais e simulados seguiram a mesma tendência.
\end{abstract}

\section{INTRODUÇÃO}

A Flotação é um método utilizado para separar e remover impurezas que se encontram em suspensão na solução, através da introdução de bolhas de ar. As espécies em suspensão se aderem às bolhas de ar formando agregados pelas partículas hidrofóbicas e, por serem menos densas que o fluido, dirigem-se à superfície (Gardusi et al., 2014).

O papel das bolhas de ar é o de coletar e transportar as partículas suspensas até a superfície, onde há a formação da espuma, da qual se faz a separação das partículas. Por isso, é de extrema importância a utilização de sistemas de aeração que possibilitem o controle do tamanho e da uniformidade das bolhas, juntamente com a utilização de tensoativos, para que se possa ter uma melhor separação através da flotação (Penna et al., 2003).

O tamanho das bolhas liberadas na mistura é controlado pelo ajuste da vazão e da pressão de emissão de ar. Bolhas com diâmetros pequenos têm uma área superficial grande, facilitando (em comparação a bolhas de volumes superiores) a maior coleta de partículas suspensas com base no mesmo volume de ar. Isso é algo de grande interesse, pois, assim, o processo de flotação é mais eficiente, tendo em vista com um menor volume de bolha a colisão bolha-partícula é melhor. Por outro lado, as bolhas de tamanho diminuto apresentam uma velocidade de ascensão baixa podendo ser inferior à velocidade descendente da polpa, o que pode levar a perdas de partículas hidrofóbicas no fluxo de material não flotado (Penna et al., 2003).

Neste contexto, avaliou-se a formação das bolhas e o efeito do tensoativo através de testes experimentais e por meio da fluidodinâmica computacional, com o software FLUENT® 18.2. 


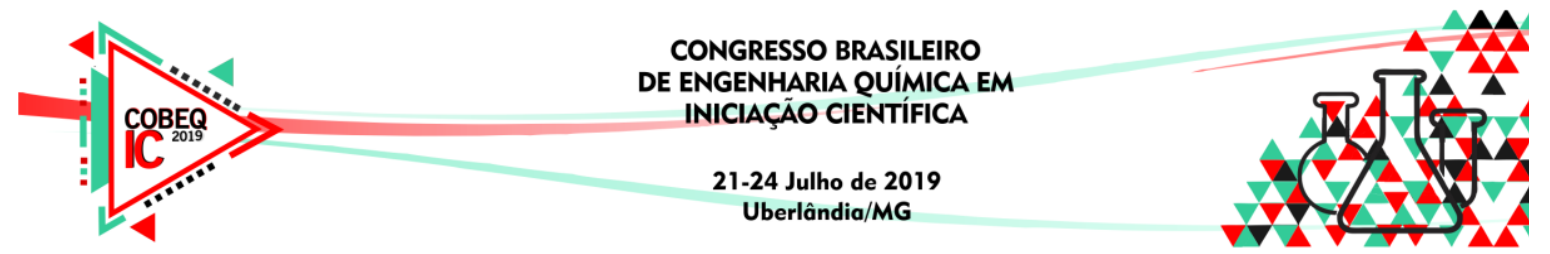

\section{MODELO MATEMÁTICO}

O modelo VOF (Volume of Fluid) pode modelar dois ou mais fluidos imiscíveis, onde uma única equação de momentum é resolvida, compartilhando o campo de velocidade entre as fases e rastreando a fração de volume de cada um dos fluidos em todo o domínio computacional. O método VOF é indicado para capturar informações sobre o comportamento da interface. A fração volumétrica $\left(\alpha_{\mathrm{k}}\right)$ de cada fase $k$ em uma célula computacional é rastreada em todo o domínio. A soma de cada fração volumétrica é igual a 1. A captura de interface é baseada na resolução do campo de fração volumétrica e, em seguida, a reconstrução da interface para cada fase $k$. As principais equações do modelo são apresentadas a seguir.

$$
\begin{aligned}
& \nabla u=0 \\
& \frac{\partial}{\partial t}(\rho \vec{u})+\nabla(\rho \vec{u} \vec{u})=-\nabla P+\nabla\left\{\mu\left[\nabla \vec{u}+(\nabla \vec{u})^{T}\right]\right\}+\rho \vec{g}+F_{S} \\
& F_{S}=\frac{\sigma\left(\rho k \nabla \alpha_{l}\right)}{0,5\left(\rho_{l}+\rho_{g}\right)} \\
& k=\nabla \hat{n} \\
& \hat{n}=\frac{n}{|n|} \\
& n=\nabla \alpha_{k} \\
& \frac{\partial}{\partial t} \alpha_{k}+\vec{u}_{k} \nabla \alpha_{k}=0 \\
& \sum_{k=1}^{2} \alpha_{k}=1 \\
& \rho(\vec{x}, t)=\alpha(\vec{x}, t) \rho_{l}+(1-\alpha(\vec{x}, t)) \rho_{g} \\
& \mu(\vec{x}, t)=\alpha(\vec{x}, t) \mu_{l}+(1-\alpha(\vec{x}, t)) \mu_{g}
\end{aligned}
$$

\section{METODOLOGIA}

A Figura 1 apresenta o aparato empregado nos testes experimentais e a geometria básica adaptada para as simulações.

Figura 1 - Aparato experimental.
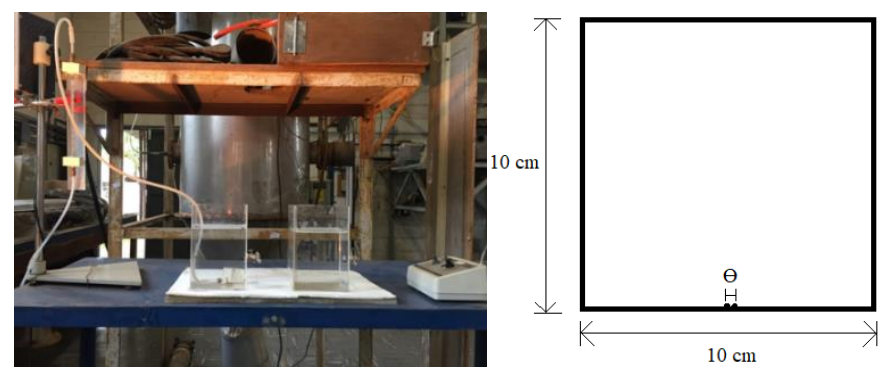


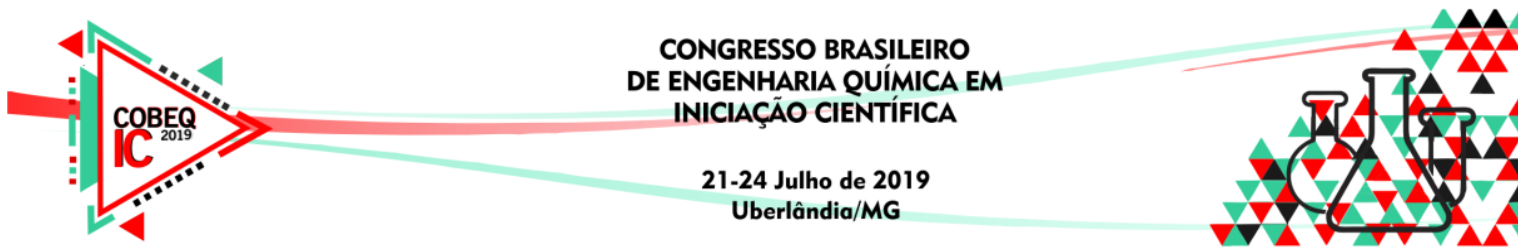

O aparato mostrado na Figura 1 é constituído por recipientes de acrílico de dimensão 12,5 x 12,5 x $13 \mathrm{~cm}$, bomba de ar elétrica e rotâmetro. Os recipientes foram preenchidos com diferentes proporções de água e etanol (tensoativo). O diâmetro do orifício de injeção de ar é inferior a um capilar, não sendo possível medi-lo com precisão. A vazão de alimentação de ar foi mantida a $27,1 \mathrm{~mL} / \mathrm{min}$. Os valores de tensão superficial para 0,20,40, 60, 80 e $100 \%$ de etanol em volume foram de $0,082,0,058,0,056,0,055,0,053$ e $0,049 \mathrm{~N} / \mathrm{m}$, respectivamente.

As malhas computacionais geradas e dados de entrada de cada simulação foram ajustados de acordo com o aparato e condições experimentais citadas acima. A geometria simulada consiste de um quadrado bidimensional de $10 \mathrm{~cm}$ de lado. O orifício de injeção de ar está localizado no centro da base. Devido ao elevado número de células geradas com o diâmetro real de orifício de entrada de $0,2 \mathrm{~mm}$, empregaram-se duas malhas computacionais com diâmetros diferentes a fim de verificar-se seu comportamento sobre a distribuição e característica das bolhas. A malha M-1 possui orifício de $1 \mathrm{~mm}$, enquanto que a malha $\mathrm{M}-2$, $0,5 \mathrm{~mm}$, contendo 20.000 e 80.000 células, respectivamente. A velocidade da injeção de ar foi calculada baseada na área de seção de entrada de cada malha, de forma a manter-se a vazão volumétrica de ar igual a $27,1 \mathrm{~mL} / \mathrm{min}$. O domínio foi preenchido com água (fase primária) e ar (fase secundária). No tempo $0,0 \mathrm{~s}$, injeta-se ar através do orifício de entrada. A velocidade de injeção de ar para a malha com orifício de diâmetro de $1 \mathrm{~mm}$ foi de $0,575 \mathrm{~m} / \mathrm{s}$, enquanto que para a de $0,5 \mathrm{~mm}$ foi de $2,3 \mathrm{~m} / \mathrm{s}$. As malhas computacionais geradas estão na Figura 2.

Figura 2 - a) malha M-1; b) malha M-2.

a)

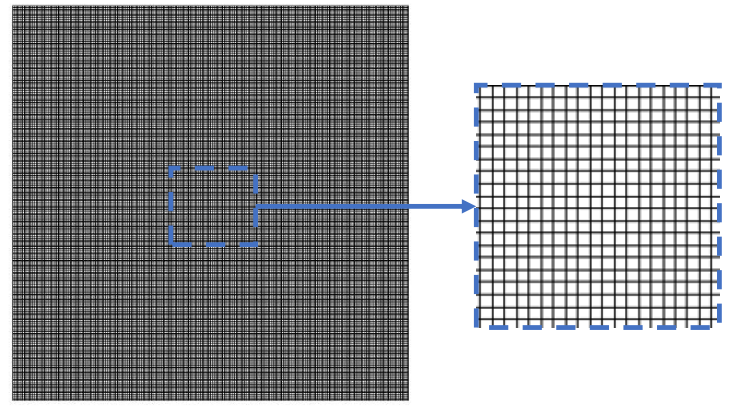

b)

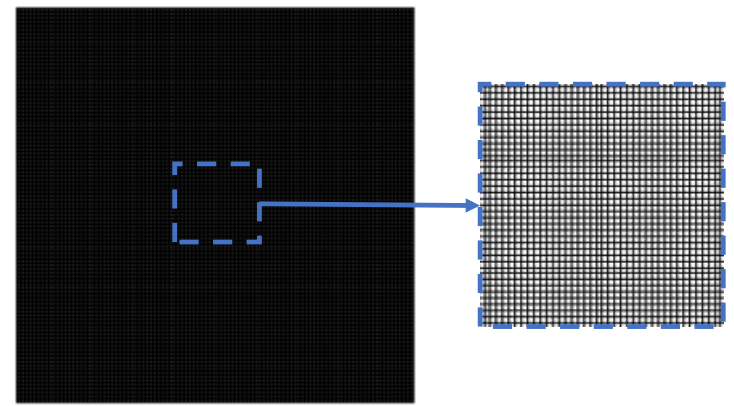

O tempo simulado para cada malha, variando-se a tensão superficial com os dados disponíveis até a obtenção dos resultados foi de $1 \mathrm{~s}$. Para inferir a velocidade das bolhas em cada simulação, ferramentas de pós-processamento disponíveis no software FLUENT foram empregadas, possibilitando a obtenção da velocidade média de bolhas na região caracterizada. Para medida do diâmetro de bolhas, para cada tensão superficial analisada, utilizou-se o software Image Pro Plus 6. Por meio do contorno de fração volumétrica de ar, calibrou-se no software a dimensão já conhecida do equipamento simulado $(10 \mathrm{~cm})$. Efetuou-se uma média aritmética entre comprimentos horizontal, vertical e de ambas diagonais de uma bolha. A média de tais comprimentos caracterizou o diâmetro da bolha de ar naquela condição.

\section{RESULTADOS E DISCUSSÃO}

Em relação a malha M-1, a Tabela 1 mostra as velocidades de bolhas de ar simuladas e medidas experimentalmente em função da tensão superficial, bem como o erro em relação à medida experimental. 


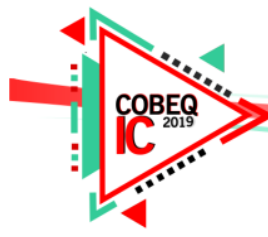

Tabela 1 - Velocidades das bolhas de ar simuladas para a malha M-1e medidas experimentalmente em função da tensão superficial da água

\begin{tabular}{|c|c|c|c|}
\hline Tensão $(\mathrm{N} / \mathrm{m})$ & $\begin{array}{c}\text { Velocidade } \\
\text { Experimental }(\mathrm{m} / \mathrm{s})\end{array}$ & $\begin{array}{c}\text { Velocidade } \\
\text { Simulada }(\mathrm{m} / \mathrm{s})\end{array}$ & Erro (\%) \\
\hline 0,082 & 0,264 & 0,273 & 3,45 \\
\hline 0,058 & 0,239 & 0,280 & 17,13 \\
\hline 0,056 & 0,220 & 0,270 & 22,50 \\
\hline 0,055 & 0,246 & 0,257 & 4,47 \\
\hline 0,053 & 0,239 & 0,263 & 10,24 \\
\hline 0,049 & 0,238 & 0,273 & 14,81 \\
\hline
\end{tabular}

Em relação a malha M-2, a Tabela 2 mostra as velocidades de bolhas de ar simuladas e medidas experimentalmente em função da tensão superficial, bem como o erro em relação à medida experimental.

Tabela 2 - Velocidades das bolhas de ar simuladas para a malha M-2 e medidas experimentalmente em função da tensão superficial da água

\begin{tabular}{|c|c|c|c|}
\hline Tensão (N/m) & $\begin{array}{c}\text { Velocidade } \\
\text { Experimental }(\mathrm{m} / \mathrm{s})\end{array}$ & $\begin{array}{c}\text { Velocidade } \\
\text { Simulada }(\mathrm{m} / \mathrm{s})\end{array}$ & Erro (\%) \\
\hline 0,082 & 0,264 & 0,297 & 12,28 \\
\hline 0,058 & 0,239 & 0,273 & 14,34 \\
\hline 0,056 & 0,220 & 0,287 & 30,07 \\
\hline 0,055 & 0,246 & 0,257 & 4,47 \\
\hline 0,053 & 0,239 & 0,297 & 24,20 \\
\hline 0,049 & 0,238 & 0,277 & 16,21 \\
\hline
\end{tabular}

Para a malha M-1, as velocidades simuladas apresentaram erro máximo de 22,50\% para as mesmas condições de tensão superficial, quando comparadas às experimentais. Em relação ao diâmetro de bolhas, os erros apresentados em relação aos dados experimentais foram maiores (máximo de $72,41 \%$ ). O diâmetro das bolhas simuladas foi maior do que as experimentais. Esta diferença de tamanhos é justificada pela maior dificuldade encontrada para a realização das simulações, a partir da geração de uma malha computacional que tivesse diâmetro de orifício de entrada de ar igual ao experimental. Enquanto o diâmetro de injeção de ar experimental é $0,2 \mathrm{~mm}$, o diâmetro de injeção da malha computacional é de $1 \mathrm{~mm}$, no caso da malha M-1.

Ressalta-se que, quanto menor for o diâmetro de injeção de ar da malha computacional, mais refinada esta será, ocasionando maior esforço computacional, consequentemente, mais tempo para a convergência das equações da continuidade e momentum.

Com o intuito de analisar a influência do diâmetro de injeção de ar sobre as grandezas de velocidade e diâmetro de bolhas, construiu-se a malha M-2. A geração de uma malha com diâmetro de entrada de ar 50\% menor acarretou no aumento de 60.000 células em relação à malha M-1. A Figura 3 mostra a comparação entre os diâmetros experimentais e simulados em função da tensão superficial do líquido para as malhas M-1 e M-2. 


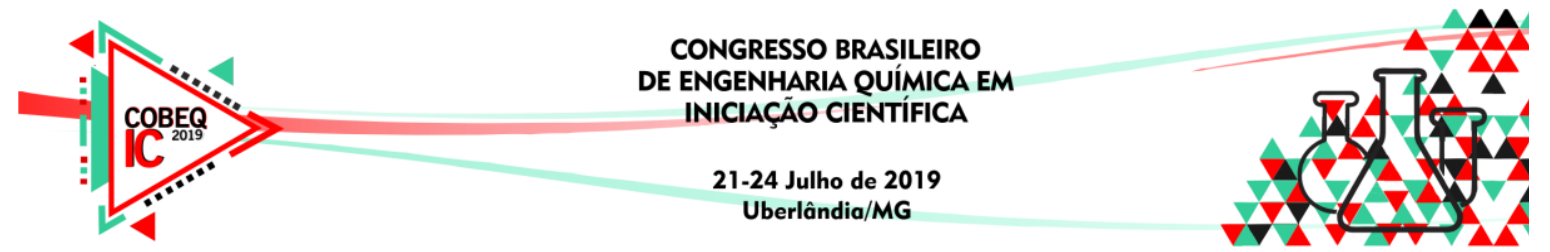

Figura 3 - Diâmetros das bolhas de ar simuladas para as malhas M-1 e M-2 medidas experimentalmente em função da tensão superficial da água.

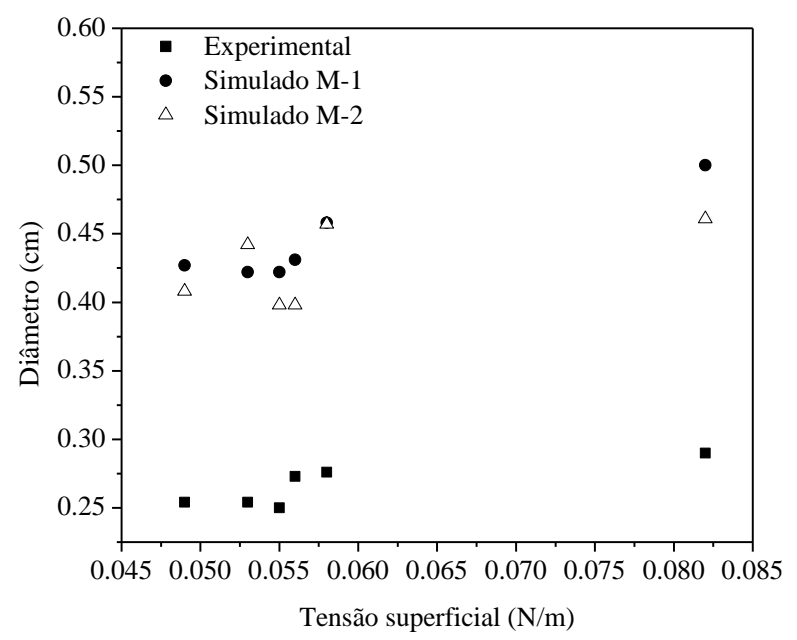

Para a malha M-2, a qual demandou consideravelmente mais tempo para a convergência das equações do modelo, o erro máximo obtido para as velocidades simuladas foi de $30,07 \%$ em relação às experimentais. Apesar deste valor ser maior do que o obtido com a malha M-1, ainda representa baixa diferença quando comparado aos dados experimentais. No quesito de diâmetro de bolhas, o erro máximo calculado foi de $74,13 \%$ em relação aos valores experimentais. Este valor é quase o mesmo que o obtido utilizando a malha M-1. Para a situação analisada, a geração de uma malha computacional mais refinada, com metade do diâmetro do orifício de injeção de ar, não é justificável, visto que os erros máximos de velocidade e diâmetro foram maiores, assim como o esforço computacional para resolução do modelo VOF.

Durante as simulações foi observado o fenômeno de coalescência das bolhas de ar formadas, conforme mostrado na Figura 4. Observa-se uma sequência de contornos instantâneos de fração volumétrica de ar, onde é possível verificar a coalescência entre duas bolhas sucessivas e uma rápida expansão da bolha formada na direção horizontal e vertical.

Figura 4 - Coalescência de bolha de ar.

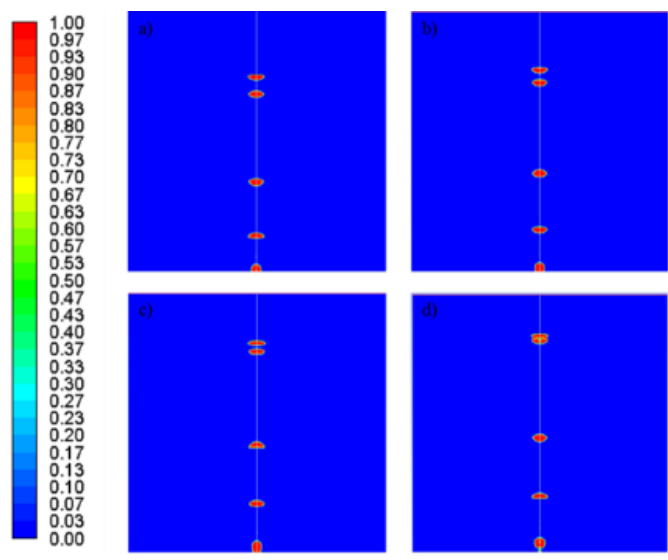




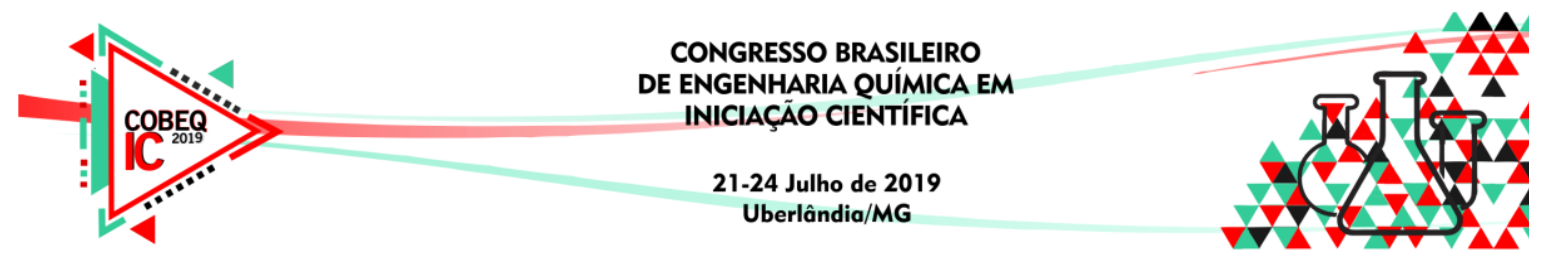

\section{CONCLUSÕES}

Tanto para a malha M-1 quanto M-2, os erros das velocidades das bolhas de ar simuladas em relação às experimentais foram aceitáveis, correspondendo a máximos de 22,50\% e 30,07\%, respectivamente. Quanto ao diâmetro das bolhas de ar, as simulações apresentaram diâmetros de bolha maiores que os experimentais, com erros máximos de $72,41 \%$ e $74,13 \%$ para as malhas M-1 e M-2, respectivamente. O perfil de diâmetro de bolhas em função das tensões superficiais seguiu a mesma tendência dos resultados experimentais.

Para o sistema estudado e simulações realizadas, deve-se optar pela malha computacional M-1, visto que os erros máximos de velocidade e diâmetro de bolhas foram menores, assim como o esforço computacional para convergência das equações da continuidade e momentum do modelo VOF. No entanto, deve-se realizar maiores investigações acerca do modelo e procedimento experimental empregado para justificar o motivo de a malha mais refinada (M2) apresentar maiores desvios.

\section{SÍMBOLOS}

$\sigma:$ coeficiente de tensão superficial [-]

$k$ : curvatura da superfície local [-]

Fs: força de tensão superficial por unidade de volume

$\hat{n}$ : superfície unitária do vetor normal [-]

\section{REFERÊNCIAS}

GARDUSI, F.; FILHO, A.M.R.; LUZ, P.H.M.; CARVALHO, G.R.L.; REIS, A.S.; BARROZO, M.A.S. Avaliação da distribuição do diâmetro de bolhas em coluna de flotação. XIX Jornada de Engenharia Química, UFU, Uberlândia (MG), 2014.

PENNA, R.; OliVEIRA, M. L. M.; VALADÃO, G. E. S.; PERES, A. E. C. Estudo comparativo entre dois sistemas de aeração de coluna de flotação. Revista Escola de Minas, v. 56, n. 3, 2003. 